\title{
Ciclo de mejora docente en la asignatura Instituciones Islámicas
}

\author{
ANTONIO CONSTÁN-NAVA \\ Departamento de Filologías Integradas \\ Universidad de Sevilla \\ aconstan@us.es \\ ORCID: https://orcid.org/0000-0003-3804-4298 \\ D.O.I.: http://dx.doi.org/10.12795/JDU.2018.i01.91 \\ Pp.: 1622-1640
}

\section{Resumen}

La comunicación presenta el diseño y desarrollo del Ciclo de Mejora Docente (CMD) para el 40 bloque Instituciones políticas. El soberano de la asignatura Instituciones Islámicas del 2o curso del Grado en Estudios Árabes e Islámicos. Un bloque en el que abundan conceptos abstractos y de difícil comprensión para un alumnado acostumbrado a ideas y datos concretos, conceptos que tradicionalmente se han explicado de manera magistral al ser una asignatura enraizada en estos estudios desde su implantación en los primeros planes de estudios.

Palabras clave: Grado Estudios Árabes e Islámicos, Instituciones Islámicas, Innovación docente, docencia universitaria, ciclo de mejora docente, metodología 


\section{Descripción del contexto de la intervención}

La asignatura elegida para el CMD ha sido Instituciones Islámicas. Es una asignatura optativa y cuatrimestral de 20 curso del Grado en Estudios Árabes e Islámicos. Consta de 6 créditos y se imparte en 3 clases semanales de 90 minutos. El programa de la asignatura, publicado en la web de la US, incluye los objetivos: apoyar el estudio y conocimiento de la lengua árabe y estudiar, conocer y comprender las diferentes instituciones que han nacido, crecido y desaparecido en la civilización islámica a lo largo de su historia, instituciones de carácter religioso, jurídico, político, administrativo, social y militar. El desarrollo del curso está dividido en los siguientes bloques temáticos (BT): I. Introducción, centrada en aspectos procedimentales y formales, así como los recursos y herramientas básicos; II. Sociedad en la Arabia preislámica, su situación política, social y económica; III. Comienzos del islam, Muhammad como líder terrenal, el Corán y los diferentes grupos de musulmanes; IV. Instituciones políticas. El soberano. La institución política del Califato, desde los califas ortodoxos hasta nuestros dias; V. Mujer y su papel en el poder, tanto en la teoría como en la práctica, desde los inicios del islam hasta nuestros días; VI. El mundo jurídico islámico; VII. La familia musulmana como institución: matrimonio, divorcio y heredad. Otras instituciones.

El curso empezó con 3 estudiantes matriculados, lo que, como docente, generó un pequeño desánimo. Sin embargo, tras la primera sesión, en la que ya iba introduciendo los cambios de los que hablaré a continuación, la asignatura terminó con 9 estudiantes matriculados, 8 de ellos han asistido con regularidad. 


\section{Diseño previo del Ciclo de Mejora Docente (CMD)}

Este es el segundo año que imparto la docencia completa de la asignatura. El primer año había usado un modelo metodológico clásico: la clase magistral, con algunas actividades prácticas basadas en trabajos de investigación sobre temáticas que los propios estudiantes tuvieran interés en conocer. El haber estado desarrollando este modelo fue más por la inercia de proceder de la misma manera que yo aprendí en mi época de estudiante, es decir, poniendo en práctica un modelo tradicional asimilado de forma pasiva. Si bien es cierto que buscaba introducir en mis sesiones algunas actividades que se alejasen de la clase magistral, como alguna proyección de vídeo o traer alguna persona experta en un aspecto de la materia, es cierto que lo que estaba haciendo era cambiar el locutor normal de la clase (yo) por otro (el narrador del vídeo o el experto traído). Esto hizo que el alumnado acabara desconectando, incluso abandonando la presencialidad, acudiendo más para solucionar dudas que para seguir un desarrollo normal de la asignatura. Por esta razón, durante el pasado verano, estuve dándole vueltas a cómo enfocar la docencia, qué podía incluir en las sesiones que rompieran la monotonía de una clase magistral y dónde podía incluir mejoras o cambios en el propio temario para que este fuera más atractivo.

Releyendo las líneas anteriores, cualquier lector se da cuenta de una cosa: el foco de cambio lo estaba centrando en mí, en cómo yo, qué yo y dónde yo. De este aspecto me di cuenta en un curso que realicé también dentro del Programa de Innovación Docente del I.C.E. La innovación en la educación superior centrada en las personas: el método ECO y terminé de convencerme en el Curso General de Docencia Universitaria. Debía cambiar el foco de atención de mí hacia el alumno, salir de mi ego docente centrado en cumplir con unos contenidos formales de enseñanza y 
enfocar el proceso en el estudiante. Por lo que replanteé estas cuestiones (Bain, 2004, 61-80) en cómo quiere aprender el estudiante, qué quiere aprender y dónde quiere poner sus esfuerzos de aprendizaje. Lógicamente, siempre dentro de los contenidos de la asignatura. El diseño del CMD de la asignatura elegida coincidió en el tiempo con el bloque temático IV. Este es el bloque central de la asignatura y, por ende, el más importante. El diseño del mapa conceptual fue el que marcó la secuencia de actividades comentadas más adelante:

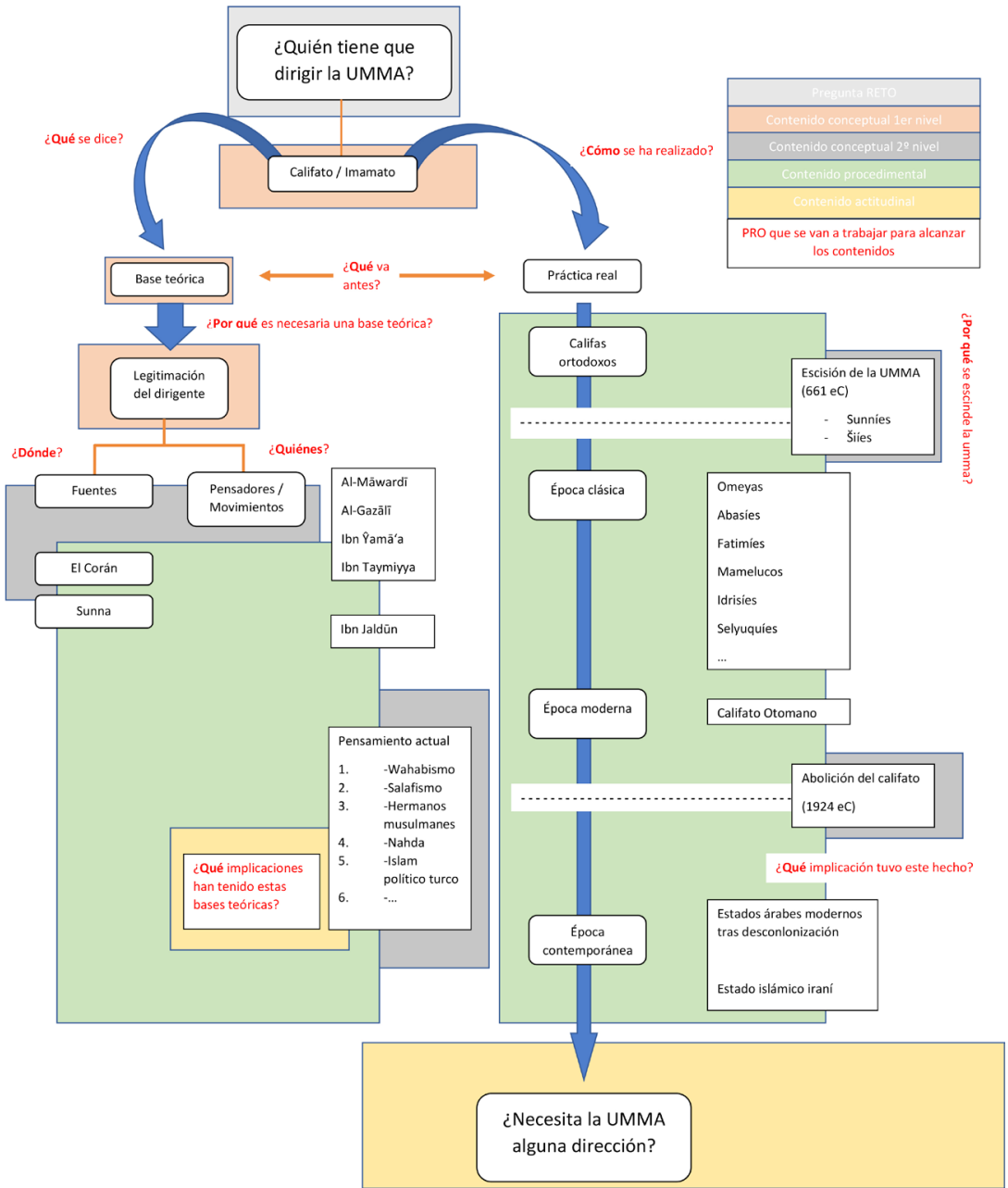

Figura 1. Mapa conceptual del BT del ciclo de Mejora Docente desarrollado

Jornadas de Formación e Innovación Docente del Profesorado | № 1 (2018)

(C) Esta obra se distribuye con la licencia Creative Commons Reconocimiento-NoComercial-SinObraDerivada Internacional (CC BY-NC-ND 4.0.) 
Para la secuencia de las actividades, el CGDU me ha guiado en el cambio del anterior modelo metodológico tradicional clase magistral hacia un modelo metodológico propio, centrado en el estudiante, que se puede resumir en el siguiente esquema:

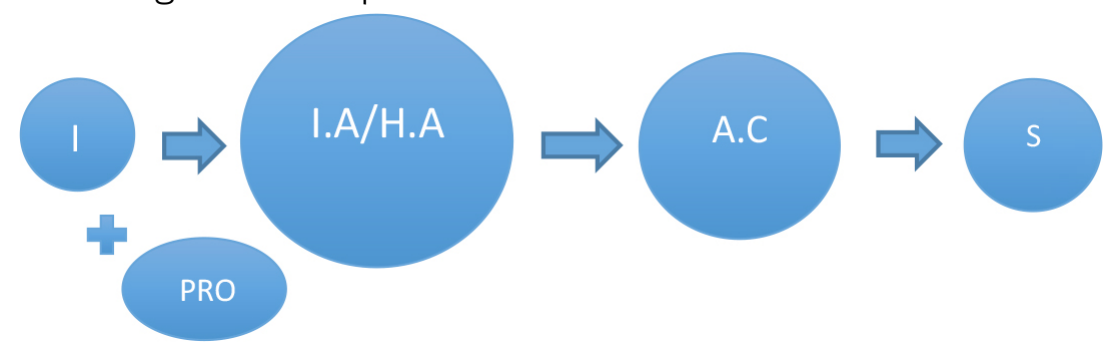

I: Introducción; PRO: Planteamiento de la problemática; I.A/H.A: Ideas/ hipótesis de los estudiantes; A.C.: Actividades de contraste; S: Síntesis

La idea incorporada era trabajar los bloques de la asignatura a través de retos, una adaptación del Design thinking ECO a este modelo metodológico. Cada una de las sesiones está planteada para que sean los estudiantes quienes realmente tengan el papel principal. La 1a sesión que abre el BT empezamos con una introducción I centrada en contextualizar y resumir de qué trata el contenido de este bloque (focalizada sobre todo en los contenidos conceptuales y procedimentales). Esta introducción sirve también para formar los grupos de trabajo, pues para el desarrollo del modelo metodológico, es imprescindible que los estudiantes trabajen en grupo. Se les da unos materiales iniciales sobre el BT y los estudiantes han de trabajar esa primera sesión en formular una pregunta-reto que les induzca en la búsqueda y creación de los contenidos del bloque durante las siguientes sesiones. En la 1a sesión, tendríamos un PRO que es buscar la cuestión-reto, y con los materiales iniciales, los estudiantes trabajarian la fase de I.A/H.A., donde observo y voy dando una secuencia de preguntas A.C. (Finkel, 2008, 153-187) para que contrasten sus ideas y les sirva de guía. En esta sesión, durante la fase de síntesis S deben tener planteada la cuestión-reto 
y darla a conocer. En el resto de las sesiones, la fase de introducción sirve para resumir el día anterior y las cuestiones clave sobre aspectos conceptuales y procedimentales abordadas, y otros inmediatos de la sesión en ciernes. En cada una de estas sesiones, en la fase de I.A/H.A., proveo a los estudiantes de materiales de trabajo, y, durante la sesión, doy una secuencia de cuestiones u otras actividades de contraste (en donde suelo incluir conceptos actitudinales) que les ayuden en la búsqueda y creación del contenido, intentando dar respuesta a las cuestiones puntuales de la sesión sin perder de vista la cuestión-reto. Antes de acabar la sesión, siempre dedicamos un tiempo para sintetizar el trabajo que han realizado. La última sesión es donde, por grupos, exponen el trabajo realizado, momento en el que han de dar respuesta a la pregunta-reto inicial a través de los contenidos conceptuales, procedimentales y actitudinales. Además, han de entregarme un breve portafolio de todo el trabajo desarrollado.

Jornadas de Formación e Innovación Docente del Profesorado | № 1 (2018) Esta obra se distribuye con la licencia Creative Commons 
Tabla 1.

Secuencia de actividades programada

1a sesión

\begin{tabular}{l|l}
1 & 1 \\
\hline
\end{tabular}

$15^{\prime}$

Introducción del BT

Se presenta y contextualiza el BT IV. El bloque engloba: Tema 7. La institución califal y el islam: califas ortodoxos. Diferentes grupos de musulmanes; Tema 8. Pirámide de la soberanía en el islam clásico y situación actual. Se hace un feed-back de los datos extraídos del cuestionario inicial realizado durante la sesión del 18 de octubre.

\begin{tabular}{|l|l|l|l|}
\hline \multicolumn{2}{|l|}{ Recursos: } & \multicolumn{2}{l|}{ Pizarra y tiza } \\
\hline 2 & PRO & $5^{\prime}$ & Planteamiento de la problemática \\
\hline
\end{tabular}

Se forman los grupos de trabajo. Se reparte un texto formado de extractos de varios periódicos digitales actuales que versan sobre la misma temática y que tienen que ver con una noticia de actualidad (el ISIS) para que las estudiantes la relacionen con el BT IV. Deben leerlo, primero, de manera individual y, en segundo lugar, siguiendo el esquema de trabajo habitual por grupos.

\begin{tabular}{|l|l|l|l|}
\hline \multicolumn{2}{|l|}{ Recursos: } & Texto de la sesión \\
\hline 3 & I.A/H.A & $20^{\prime}$ & $\begin{array}{l}\text { Trabajo grupal sobre el material } \\
\text { textual }\end{array}$ \\
\hline
\end{tabular}

En grupo, los estudiantes han de ir entresacando aquellos conceptos que tengan que ver con el BT. Algunos de los conceptos ya se visto a lo largo de las sesiones precedentes; otros, es la primera vez que aparecen. Han de ir confeccionando un listado provisional de aquello que creen relacionar con el BT.

\begin{tabular}{|l|l|l|l|}
\hline \multicolumn{2}{|l|}{ Recursos: } & Texto de la sesión, internet \\
\hline 4 & C.A & $15^{\prime}$ & Preguntas disruptivas \\
\hline
\end{tabular}

Se les ofrecerá a los estudiantes 2 preguntas-tipo sobre el cuestionario inicial, pero que versarán directamente sobre el contenido del texto dado. Han de contrastar la respuesta a las preguntas con las listas que ellos mismos están confeccionando.

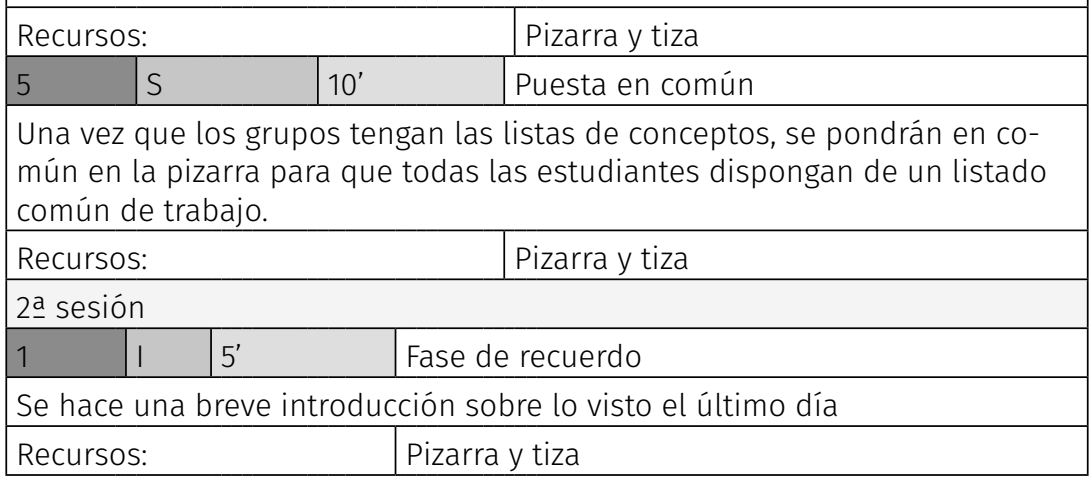

Jornadas de Formación e Innovación Docente del Profesorado I № 1 (2018) Esta obra se distribuye con la licencia Creative Commons Reconocimiento-NoComercial-SinObraDerivada Internacional (CC BY-NC-ND 4.0.) 


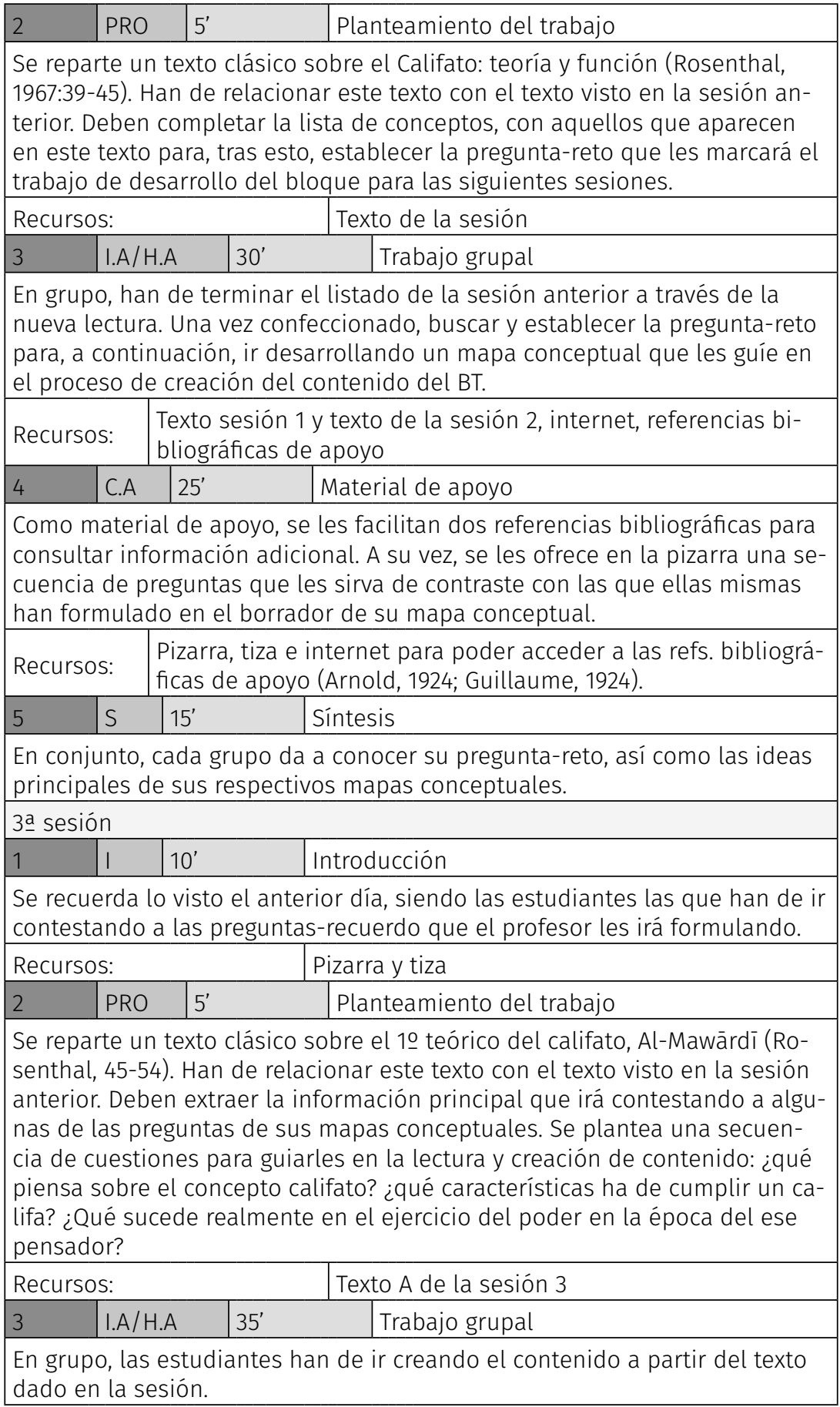

Jornadas de Formación e Innovación Docente del Profesorado | № 1 (2018) Esta obra se distribuye con la licencia Creative Commons Reconocimiento-NoComercial-SinObraDerivada Internacional (CC BY-NC-ND 4.0.) 


\begin{tabular}{|l|l|l|l|l|}
\hline \multicolumn{2}{|l|}{ Recursos: } & Texto mencionado \\
\hline 4 & C.A & $25^{\prime}$ & Material de contraste \\
\hline
\end{tabular}

Se les da un 20 texto, esta vez, de otro de los pensadores clásicos del califato, Al-Gazālī (Rosenthal, 54-59), que han de comparar con los datos extraídos del anterior texto.

Recursos:

\begin{tabular}{|l|l|l|l|}
\hline 5 & S & $15^{\prime}$ & Sintesis \\
\hline
\end{tabular}

Las estudiantes han de tener elaborado parte del contenido de su mapa conceptual que vaya respondiendo a la pregunta-reto a partir de estos 2 primeros pensadores

4a sesión

\begin{tabular}{|l|l|l|l|}
\hline 1 & 1 & $15^{\prime}$ & Introducción \\
\hline
\end{tabular}

Se recuerda lo visto el anterior día, siendo las estudiantes las que han de ir contestando a las preguntas-recuerdo que el profesor les irá formulando al respecto del pensamiento sobre el califato de los 2 pensadores clásicos vistos.

\begin{tabular}{l|l}
\hline Recursos: & Pizarra y tiza
\end{tabular}

\begin{tabular}{l|l|l|l|}
\hline 2 & PRO & $5^{\prime}$ & Planteamiento del trabajo \\
\hline
\end{tabular}

Se reparte un texto clásico sobre el tercer pensador del califato, Ibn Ŷamā‘a (Rosenthal, 59-66). Han de relacionar este texto con los pensadores vistos en la sesión anterior. Deben ir extrayendo la información principal que irá contestando a algunas de las preguntas de sus mapas conceptuales. Les replanteo la misma secuencia de cuestiones de la sesión anterior.

\begin{tabular}{|l|l|l|l|}
\hline \multicolumn{2}{|l|}{ Recursos: } & \multicolumn{2}{l|}{ Texto A de la sesión 4} \\
\hline 3 & I.A/H.A & $35^{\prime}$ & Trabajo grupal \\
\hline
\end{tabular}

En grupo, las estudiantes han de ir creando el contenido a partir del texto dado en la sesión.

\begin{tabular}{l|l|l}
\hline Recursos: & Texto mencionado \\
\hline 4 & C.A & $20^{\prime}$
\end{tabular}

\begin{tabular}{|l|l|l|l|}
\hline 4 & C.A & $20^{\prime}$ & Material de contraste \\
\hline
\end{tabular}

Se les da un 2o- texto, esta vez, del último de los pensadores clásicos del califato, Ibn Taimiyya (Rosenthal, 66-75), que han de comparar con los datos extraídos del anterior texto.

\begin{tabular}{|c|c|c|c|}
\hline \multicolumn{3}{|c|}{ Recursos: } & Texto B de la sesión 4 \\
\hline 5 & $S$ & $15^{\prime}$ & Sintesis \\
\hline \multicolumn{4}{|c|}{$\begin{array}{l}\text { Las estudiantes han de tener elaborado parte del contenido de su mapa } \\
\text { conceptual que vaya respondiendo a la pregunta-reto a partir de estos } 2 \\
\text { primeros pensadores. }\end{array}$} \\
\hline \multicolumn{4}{|c|}{ 5á sesión } \\
\hline 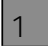 & & $15^{\prime}$ & Introducción \\
\hline
\end{tabular}


Se recuerda lo visto el anterior día, siendo las estudiantes las que han de ir contestando a las preguntas-recuerdo que el profesor les irá formulando al respecto del pensamiento, en esta ocasión, recapitulando los 4 pensadores trabajados.

\begin{tabular}{l|l}
\hline Recursos: & Pizarra y tiza
\end{tabular}

\begin{tabular}{l|l|l|}
2 & PRO & 5
\end{tabular}

Se reparte un texto clásico sobre el gran pensador del califato, Ibn Jaldún (Rosenthal, 99-127). Han de relacionar este autor con los pensadores vistos para cerrar el contenido del BT. Deben extraer la información principal que irá contestando a las preguntas de sus mapas conceptuales. Les vuelvo a plantear la misma secuencia de cuestiones.

\begin{tabular}{l|l}
\hline Recursos: & Texto de la sesión 5 \\
\hline 3
\end{tabular}

\begin{tabular}{l|l|l|}
\hline 3 & I.A/H.A & 35 \\
\hline
\end{tabular}

En grupo, las estudiantes han de ir creando el contenido a partir del texto dado en la sesión.

\begin{tabular}{|l|l|l|l}
\hline 4 & C.A & $20^{\prime}$ & Material de contraste \\
\hline
\end{tabular}

Se pone un fragmento del vídeo El fin del Imperio Otomano - El Medio

Oriente fragmentado y una noticia sobre la abolición del califato otomano en 1924, hasta nuestros días. Han de relacionar esta nueva información con todos los datos extraídos durante las sesiones.

Recursos:

Ordenador, internet, proyector, altavoces y texto mencionado

\begin{tabular}{l|l|l|l|}
\hline 5 & S & $15^{\prime}$ & Sintesis \\
\hline
\end{tabular}

Las estudiantes han de tener elaborado completamente el contenido de su mapa conceptual, respondiendo a la pregunta-reto a partir de estos 2 primeros pensadores

6a sesión

\begin{tabular}{|l|l|l} 
Ev & 50 & Exposición grupal
\end{tabular}

$20^{\prime}-25^{\prime}$ para exponer las conclusiones del trabajo realizado durante las sesiones precedentes.

\begin{tabular}{|l|l|l|l}
\hline \multicolumn{2}{|l|}{ Recursos: } & Pizarra, tiza, proyector, altavoces. \\
\hline 2 & Ev & $40^{\prime}$ & Cuestionario final
\end{tabular}

Se les pasará el mismo cuestionario que se les hizo al inicio, para comprobar si el trabajo de las sesiones precedentes y escuchar el trabajo del otro grupo, ha incidido en su propias valoraciones y opiniones al respecto de las preguntas que se formulan.

\begin{tabular}{l|l}
\hline Recursos: & Cuestionario \\
\hline
\end{tabular}

Introduje en el CMD un cuestionario inicial (Porlán, 2017:77 y ss) para ver qué ideas previas tenían los estudiantes sobre los contenidos del BT contenidos en mi mapa

Jornadas de Formación e Innovación Docente del Profesorado I № 1 (2018) Esta obra se distribuye con la licencia Creative Commons Reconocimiento-NoComercial-SinObraDerivada Internacional (CC BY-NC-ND 4.0.) 
conceptual (figura 1), cuyo análisis me serviría para diseñar las escaleras de aprendizaje (figuras 3, 4, 5 y 6) y que sirvieron para rediseñar las actividades de aprendizaje propuestas. Este cuestionario se pasaría al final del del CMD, con estas cuestiones: 1) ¿Cómo crees que sería la organización politica del islam si Muhammad hubiese dejado establecida su sucesión, sabiendo que no tuvo herederos varones? Trata de dar tu opinión con el máximo de concreción posible; 2) ¿Piensas que es necesario que alguien dirija a los creyentes de una comunidad religiosa, en este caso, la islámica? Argumenta detalladamente tu respuesta.

\section{Aplicación del Ciclo de Mejora Docente}

La 1á sesión no se desarrolló como esperaba debido a que solo asistieron 4 alumnas y un oyente. Con todo, la clase se planteó tal y como se había planificado. Se presentó y contextualizó el BT IV. Al mismo tiempo, se transmitieron al alumnado algunas apreciaciones sobre el cuestionario inicial. Se repartió el primer texto y se dieron las instrucciones de trabajo para todo el BT: formar 2 grupos de trabajo de 4 miembros cada uno. Constituyeron los mismos grupos que vienen formando desde el primer día de clase. Esto hizo que uno empezara con 1 miembro y el otro con 3. El alumno oyente se puso con la estudiante que estaba sola. Trabajaron, primero, de forma individual: leyeron el texto una primera vez para saber de qué iba. Después, se pusieron a trabajar en grupo. Durante unos 20 minutos, confeccionaron listas de conceptos extraídos del texto que tuvieran que ver con el contenido del BT (algunos ya se habían planteado anteriormente, sin ahondar en ellos). Mientras, iba observando ambos grupos, formulándoles cuestiones sobre el propio texto. El grupo con menos miembros fue el que más dificultades tuvo, pues la estudiante confió en el alumno oyente y, en vez de buscar conceptos, estaban extrayendo argumentos y alguna 
descripción descontextualizada. En la etapa de S, se creó una clasificación inicial de conceptos.

En la 2a sesión se retomó el corpus conceptual del final de la sesión anterior. Hubo nuevamente 3 estudiantes: 1 de ellas no estuvo en la sesión anterior, y faltaron 2 que sí estuvieron. Tampoco vino el alumno oyente. Se planteó la PRO: los grupos trabajaron en la pregunta-reto que les habría de guiar en el proceso de estudio y creación del contenido del BT. Completaron el corpus conceptual comenzado anteriormente. Tras 25 minutos de I.A/H.A., les formulé una secuencia de preguntas: ¿por qué se titula así el texto? ¿Qué conceptos en árabe se dan en el mismo? ¿Por qué esos conceptos y no otros? ¿Quién van a tratar de responden esos conceptos? ¿En qué épocas? Estas preguntas centraron la búsqueda de la pregunta-reto. Los últimos 10 minutos de clase, se ha plasmado en la pizarra la información trabajada y ambos grupos llegaron a una misma pregunta-reto: ¿Quién debe dirigir la comunidad islámica?

En la 3a sesión se recordó lo que llevábamos de proceso de construcción de contenido del BT. Trabajaron en la elaboración de un mapa conceptual que les facilitara y les fuera guiando en el proceso de creación del contenido. A su vez, se les entregó el texto A de la sesión. De este texto, extrajeron la información que les fuera ayudando en contestar a algunas de las preguntas de sus mapas conceptuales. Les planteé la siguiente secuencia de cuestiones: ¿qué piensa el autor sobre el concepto califato? ¿Qué características ha de cumplir un califa? ¿Qué sucede realmente en el ejercicio del poder en la época del ese pensador? Se les dio el texto B de la sesión (A.C.), para su comparación y realización del mismo proceso. La clase terminó sin llegar a trabajar todo el 2o texto, por lo que no se sintetizó de manera completa el trabajo previsto. Otro problema continuó siendo el mismo: solo asistieron 4 estudiantes, lo que dificultó el trabajo grupal y el desarrollo de las actividades previstas. 
Se retomó la 4a sesión con un recuerdo de lo realizado la sesión precedente. Solo vinieron a clase 2 alumnas: ambas del mismo grupo. Una de ellas es la que había venido regularmente y la otra se incorporaba ese día tras una enfermedad. Tenía los materiales leídos -ya que disponía de ellos en la Enseñanza Virtual-, pero se ha empleado un tiempo para situarla en el contexto del trabajo realizado y lo que se requería para esta sesión. Trabajaron donde se habían quedado: finalizando la A.C. con el 2o texto. Veinte minutos después, siguiendo el plan previsto, se les ha dado los textos A y B de la sesión para que repitieran el mismo proceso. Al ir con retraso, el trabajo fue un fracaso: solo dio tiempo de terminar la S de la sesión anterior y empezar a responder la secuencia de preguntas de la sesión anterior.

En la 5a sesión estuvo la clase al completo y formaron los grupos correctamente. Las estudiantes ausentes demostraron durante la sesión que no estaban tan perdidas como en un principio había supuesto, pues han estado trabajando los materiales fuera del horario de clase. La sesión se dedicó en retomar las actividades programadas para la sesión anterior y, por consenso, la sesión proyectada para esa sesión se aplazó al día siguiente, y la sesión del día siguiente se retrasó un día. Sin embargo, uno de los grupos me pidió exponer al día siguiente. Han estado trabajando en los dos textos entregados la sesión anterior, contestando la secuencia de preguntas para luego ponerlas en comparación con los autores vistos. Como A.C., he puesto una imagen de la pirámide de la soberanía clásica, para terminar de contrastar las ideas que han ido trabajando y desarrollando. Por grupos, han sintetizado la información hasta donde llevaban visto.

La 6á sesión se desarrolló en 2 partes: por un lado, la exposición del primer grupo. Los 4 miembros realizaron una exposición muy completa en lo que se refiere a conceptualización, terminología en árabe, así como en las 
síntesis de los materiales vistos. Su discurso lo han ido reflejando en la pizarra con un esquema diseñado conforme avanzaban en su exposición, esquema basado en el mapa conceptual desarrollado a partir de la pregunta-reto. Si bien en el desarrollo de su discurso solo se han centrado en tres de los cinco autores trabajados. Introduje una variable que no tenía prevista en el diseño del 2o CMD: tras la exposición, he pedido a los ponentes que se autoevaluaran, tanto el trabajo en grupo como el trabajo individual. A su vez, he pedido a los estudiantes oyentes que también valoraran la exposición del grupo que había expuesto. Estas valoraciones las han hecho de manera confidencial. Por otro lado, tras la exposición, el otro grupo ha seguido trabajando, centrado en recapitular y ordenar toda la información con respecto a los autores vistos, para unir esta información con el corpus conceptual extraído y clasificado en las 2 primeras sesiones del BT.

La 7a sesión extra ha sido la exposición del 2o grupo. Reflejaron previamente en la pizarra un esquema basado en su mapa conceptual. También desarrollaron la información, pero este grupo fue el que más miembros han faltado a clase, cosa que se puso de manifiesto en la falta de concreción de alguno de los contenidos del BT. Únicamente la estudiante que había trabajado las actividades desde el primer día ha demostrado tener asimilados los contenidos. Al término de la exposición, les he pasado el cuestionario final.

\section{Escalera de aprendizaje y evaluación}

La sesión previa a la implantación del CMD pasé a las estudiantes un cuestionario previo. Solo 4 estudiantes estuvieron presentes, por lo que, a pesar de ser un número escueto, no deja de ser la mitad de la clase. 
Cuestionario inicial

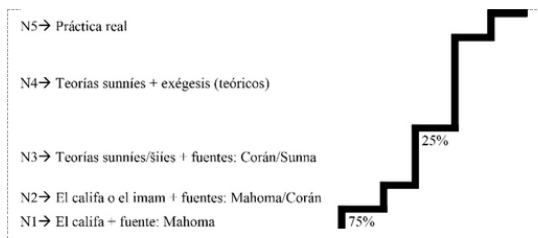

N4 $\rightarrow$ Es una cuestión, a dia de hoy $-y$ desde la muerte de
Mahoma-, irresoluble y conflictiva

$\mathrm{N} 3 \rightarrow \mathrm{El}$ Corán es la guia y no hace falta dirigente

$\mathrm{N} 2 \rightarrow$ Un único dirigente

$\mathrm{NI} \rightarrow$ Varios dirigentes

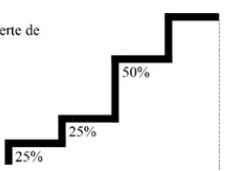

Figura 4. Escalera de aprendizaje pregunta 2

Figura 3. Escalera de aprendizaje pregunta 1

Cuestionario final
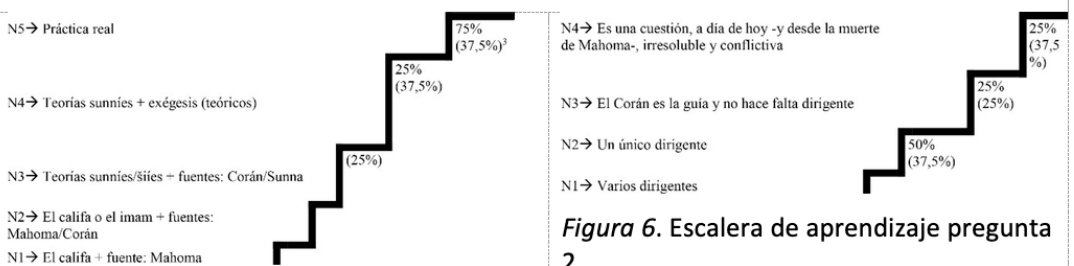

Figura 6. Escalera de aprendizaje pregunta

Figura 5. Escalera de aprendizaje pregunta 1 2

Quedando el progreso del proceso de enseñanza-aprendizaje evaluado según las siguientes tablas extraídas a partir de las escaleras iniciales y finales:

Tabla 2.

Progreso de los estudiantes que realizaron cuestionario inicial y final

\begin{tabular}{|c|c|c|c|c|}
\hline & \multicolumn{2}{|c|}{ Pregunta A (N1 $\rightarrow$ N5) } & \multicolumn{2}{c|}{ Pregunta B (N1 $\rightarrow$ N4) } \\
\hline Estudiante & Inicial & Final & Inicial & Final \\
\hline A & N1 & N5 & N1 & N2 \\
\hline B & N1 & N4 & N2 & N2 \\
\hline C & N1 & N4-N5 & N3 & N3 \\
\hline D & N3 & N5 & N3 & N4 \\
\hline
\end{tabular}

Jornadas de Formación e Innovación Docente del Profesorado | № 1 (2018)

cc) (i) $(2)$ Esta obra se distribuye con la licencia Creative Commons 
Tabla 3.

Progreso de los estudiantes que solo realizaron cuestionario final

\begin{tabular}{|c|c|c|c|c|}
\hline & \multicolumn{2}{|c|}{ Pregunta A } & \multicolumn{2}{c|}{ Pregunta B } \\
\hline Estudiante & Inicial & Final & Inicial & Final \\
\hline E & - & N3 & - & N2 \\
\hline F & - & N4 & - & N3 \\
\hline G & - & N4 & - & N4 \\
\hline H & - & N3 & - & N4 \\
\hline D & $\begin{array}{c}\text { No } \\
\text { presentada }\end{array}$ & & & \\
\hline
\end{tabular}

En general, estoy satisfecho con el resultado del CMD. Si bien con la 2a cuestión, el grado de consecución no es el esperado, tampoco puedo obviar que es la pregunta de contenido actitudinal donde entra la opinión personal (p.e., el caso de B quien dice reafirmar su criterio). En cuanto a la 1a pregunta, las estudiantes que he situado en N3 han sido aquellas que faltaron varias sesiones y no trabajaron de manera normal junto al resto de compañeras. En cuanto a C, he marcado N4-N5 ya que durante las exposiciones sí estaba en N5 pero en el cuestionario se quedó en N4, aunque para el cómputo porcentual en la escalera de aprendizaje final la he incluido en N5.

\section{Evaluación del diseño del CMD}

En primer lugar, me centraré en aquellos aspectos que considero como factor de fracaso a la hora de llevar a cabo el CMD, y que revisaré para años posteriores. Sin que sirva de excusa, el BT que ha coincidido con el CMD es el más arduo de toda la asignatura, ya que se mezclan ideas concretas con ideas formales. Esto ha supuesto una dificultad añadida a la hora de gestionar los contenidos. Así, 1) configurar las clases para que el desarrollo sea en trabajo 
grupal ha puesto de manifiesto el primer obstáculo: si los estudiantes no vienen a clase se dificulta y/o imposibilita un trabajo cooperativo satisfactorio; 2) Los textos ofrecidos como materiales, guiados por las A.C., han sido muy largos para trabajar en el tiempo de clase, por lo que estos textos serán revisados y concretizados; 3) El número de sesiones ha sido muy ajustado, y considero que una sesión añadida habría sido clave para un mejor desarrollo de las actividades; y, 4) Incluiré en las actividades programadas otras tareas complementarias y puntuables para casa, que luego se expongan en clase en una etapa de ofrecimiento del trabajo opcional para que el resto de los compañeros se haga eco de la información que aporten.

En segundo lugar, es necesario apuntar los aspectos positivos de este CMD. Por lo que, 1) si la ausencia de estudiantes dificultó el trabajo grupal, cuando los grupos estaban completos entre el 80-100\%, el desarrollo de las actividades fue fructífero, como así lo demostraron las exposiciones y el cuestionario final; 2) Creo que es un punto a favor tanto del diseño de las actividades, así como de los textos seleccionados y las consiguientes actividades el que los estudiantes partieran de la pregunta-reto formulada en mi mapa conceptual (figura 1), solo a través de las 2 sesiones primeras en las que habían de planteársela,; 3 ) los esquemas o mapas conceptuales realizados por las estudiantes se asemejan mucho al mapa conceptual del BT (figura 1); 4) La mayor parte del contenido conceptual del BT ha sido abordado por las estudiantes. Además, los contenidos procedimentales, tanto instrumentales como psicológicos, también han sido trabajados. Y los contenidos actitudinales han sido desarrollados y afianzados (figura $6)$; y, 5) La inclusión de las autoevaluaciones de los estudiantes han servido para que ellos mismos recapaciten sobre su trabajo realizado (y conseguido), lo que puede redundar en una mayor participación del estudiante en este tipo de actividades diseñadas y a mí me ayuda a evaluar mi propia docencia (Bain, 181-191). 


\section{Conclusiones}

He presentado el diseño y desarrollo del CMD para el IV BT de la asignatura Instituciones Islámicas del 2o curso del Grado en Estudios Árabes e Islámicos. Un bloque en el que abundan los conceptos conceptuales abstractos y de difícil comprensión para un alumnado acostumbrado a ideas y datos concretos, conceptos que tradicionalmente se han venido explicando de manera magistral al ser una asignatura enraizada en estos estudios desde su implantación en los primeros planes de estudios en España.

Después de repensar en cómo se han desarrollado las sesiones, soy consciente y estoy convencido de que este es el camino que se ha de tomar, alejándonos - no poco a poco- de ese magisterio tradicional, siempre bajo el ensayo-error-corrección de las actividades que se prevean en el diseño metodológico propuesto e implementado desde el primer día de clase, y que he plasmado en el CMD. Si bien existen muchas variables que pueden hacer fracasar el proceso de enseñanza-aprendizaje diseñado, también es cierto que el modelo tradicional tiene una tasa ampliamente estudiada de fracaso, con lo que, entre lo malo conocido y lo bueno por conocer, me quedo con lo segundo pues lleva implícito un adjetivo, bueno.

Jornadas de Formación e Innovación Docente del Profesorado | № 1 (2018) Esta obra se distribuye con la licencia Creative Commons 


\section{Referencias bibliográficas}

Arnold, Sir Thomas W. (1924). The Califate. Oxford: Claredon Press.

Bain, K. (2004). Lo que hacen los mejores profesores de universidad. Valencia: Publicaciones Universitat de Valencia.

Finkel, D. (2008). Dar clase con la boca cerrada. Valencia: Publicaciones Universitat de Valencia.

Guillaume, Alfred (1924). The Traditions of Islam. An Introduction to the Study of the Hadith Literature.

Porlán, R. Coord. (2017). Enseñanza universitaria. Cómo mejorarla. Madrid: Morata.

Rosenthal, Erwin I. J. (1967). El pensamiento político en el islam medieval. Madrid: Revista de Occidente.

Jornadas de Formación e Innovación Docente del Profesorado | № 1 (2018)

(c) (i) $\Theta$ Esta obra se distribuye con la licencia Creative Commons Reconocimiento-NoComercial-SinObraDerivada $\quad 4.0$ Internacional (CC BY-NC-ND 4.0.) 